\title{
Cabanyal Portes Obertes: se acabó, ¿Y ahora qué? Prácticas artísticas POLÍTICAS Y COLABORATIVAS EN LA CIUDAD
}

\author{
Cabanyal Portes Obertes: it's Over, So Now What? Policies \\ and Collaborative Artistic Practices in the City
}

\section{Emilio José Martínez Arroyo \\ Universidad Politécnica de Valencia, España.}

RESUMEN: El evento de arte Cabanyal Portes Obertes ha venido desarrollándose ininterrumpidamente desde 1998 hasta 2015 para revindicar la rehabilitación del barrio del Cabanyal de Valencia, convirtiéndose en una referencia de arte como herramienta al servicio del movimiento ciudadano en la visibilización de sus reivindicaciones. En 2015, fruto del movimiento ciudadano se consigue la retirada del proyecto de destrucción del Cabanyal y el inicio de su rehabilitación, en este momento Cabanyal Portes Obertes siente que sus objetivos han sido cumplidos y por lo tanto plantea su desaparición como evento artístico. La nueva situación política nos obliga a reflexionar como artistas cuáles pueden ser las formas más útiles que pueden adoptar las propuestas artísticas para seguir apoyando las reivindicaciones ciudadanas en el nuevo contexto del Cabanyal. Surgen así Cabanyal Arxiu Viu y CraftCabanyal dos proyectos artísticos colaborativos que acompañarán al movimiento ciudadano frente a los retos que aparecen en esta nueva situación.

Palabras Clave: arte público, movimiento ciudadano, arte político, arte colaborativo, Cabanyal.

RESUM: L'esdeveniment d'art Cabanyal Portes Obertes s'ha desenvolupat ininterrompudament des de 1998 fins a 2015 per a reivindicar la rehabilitació del barri del Cabanyal de València, $i$ es va convertir en una referència d'art com a eina al servei del moviment ciutadà en la visibilització de les seues 
reivindicacions. En 2015, fruit del moviment ciutadà, s'aconsegueix la retirada del projecte de destrucció del Cabanyal i l'inici de la seua rehabilitació, en aquest moment Cabanyal Portes Obertes sent que els seus objectius s'han complit i, per tant, planteja la seua desaparició com a esdeveniment artístic. La nova situació política ens obliga a reflexionar, com a artistes, quines poden ser les formes més útils que poden adoptar les propostes artístiques per a seguir donant suport a les reivindicacions ciutadanes en el nou context del Cabanyal. Sorgeixen així Cabanyal Arxiu Viu i CraftCabanyal dos projectes artístics col·laboratius que acompanyaran al moviment ciutadà enfront dels reptes que apareixen en aquesta nova situació.

Paraules clau: art públic, moviment ciutadà, art polític, art col·laboratiu, Cabanyal.

ABSTRACT: The art event Cabanyal Portes Obertes (Cabanyal Open Doors) evolved steadily from 1998 to 2015 as part of the campaign calling for renovation of the district of Cabanyal in Valencia. Over the years it has become a reference for art as a tool to serve citizens' movements by making their demands visible. In 2015, as a result of the citizens' movement the proposal to demolish Cabanyal was withdrawn and renovation has now begun. At this time Cabanyal Portes Obertes feels that its objectives have been met and therefore proposes its demise as an artistic event. The new political situation forces us, as artists, to reflect on the most useful ways for art to further support citizens' demands in the new Cabanyal context. These reflections have spawned Cabanyal Arxiu Viu and CraftCabanyal, two collaborative art projects that will accompany the citizens' movement in the challenges they face in this new situation.

KeYwords: public art, citizen movement, political art, collaborative art, Cabanyal. 
$\mathrm{D}$ espués de 17 años ininterrumpidos, por fin podemos celebrar que Cabanyal Portes Obertes no volverá a convocarse. Para el que a estas alturas no lo conozca, Cabanyal Portes Obertes es un evento de arte que se ha venido desarrollando en el barrio del Cabanyal de Valencia, desde 1998 hasta la actualidad, para reivindicar su rehabilitación frente a los planes destructivos del Ayuntamiento de la ciudad, gobernada por el partido popular en los últimos 24 años.

A diferencia de cualquier evento artístico habitual, en Cabanyal Portes Obertes siempre manifestamos el deseo de desaparecer, que el carácter reivindicativo del evento dejara de tener sentido en el momento que los objetivos del mismo fueran completados. Cabanyal Portes Obertes tenía como objetivo señalar la necesidad de defender el histórico barrio del Cabanyal de la destrucción planeada por las autoridades políticas, con el tiempo se convirtió también en un espacio para reflexionar sobre la ciudad, el modelo de desarrollo propuesto por la ideología neoliberal dominante, por una clase política provinciana que con el tiempo ha demostrado su inoperancia.

Esta dimensión política del evento - que no politizada puesto que no respondía a ninguna estrategia partidista, sino que surgía del propio movimiento ciudadano - ha sido determinante en la definición del propio evento, las obras y autores participantes, pero especialmente en la relación entre los dispositivos expositivos - las casas y calles del barrio - con el público asistente, generalmente muy ajeno al seguimiento de la actualidad artística en general.

Un elemento significativo de Portes Obertes ha sido su capacidad de autogestión. Cómo organizar un evento en más de una veintena de espacios expositivos y con intervenciones en el espacio público a lo largo del barrio. Debemos reconocer que la experiencia de haber organizado Movimiento-Inercia en 1996 fue determinante, proyecto desarrollado dentro del programa Kaleidoscope de la Comisión Europea, uno de los primeros programas de la Unión Europea para el desarrollo de programas culturales de colaboración entre entidades de diversos países. Desarrollado por la ASBL Les Brasseurs (Liege) en colaboración con ICA Proton (Amsterdam), Ludwin Forum Aachen y el Departamento de Escultura de la UPV (Universitat Politècnica de València), se desarrolló entre 1994-1996 con eventos en distintos espacios culturales europeos, hasta la celebración en Valencia de un «encuentro europeo de 
artistas, grupos y colectivos de $\operatorname{artistas}^{1}{ }^{1}$ que reunió a un centenar largo de artistas y colectivos de toda Europa en torno a unas jornadas y exposiciones en los que se abordaba «lo colectivo», grupos y asociaciones de artistas y ciudadanos, como herramienta para enfrentar el cambio de paradigma de la producción cultural contemporánea.

La experiencia de Movimiento-Inercia nos permitió plantear Cabanyal Portes Obertes como un evento ambicioso que tenía presente las nuevas formas de producción cultural que habíamos podido conocer, y poner a prueba. En aquel momento, finales de 1998, en la Plataforma Salvem el Cabanyal, iniciábamos nuestras primeras reuniones para enfrentar el proyecto municipal en la sede de la Asociación de Vecinos del Cabanyal - Canyamelar. La presentación de la propuesta para celebrar Cabanyal Portes Obertes fue recibida con un gran entusiasmo por parte de los vecinos y con un total desconocimiento. Lo habitual en el contexto de las asociaciones de vecinos era celebrar las semanas culturales con un carácter más local y convencional, por lo que tuvimos que dejar muy claro desde el principio que el proyecto tenía un carácter radicalmente contemporáneo, interdisciplinar y de exploración de nuevos lenguajes a partir del compromiso de artistas e intelectuales en la defensa del Cabanyal. En paralelo, la plataforma fue adquiriendo un carácter independiente de la propia asociación de vecinos para poder emprender un trabajo más específico y activista respecto a la problemática urbanística, convirtiéndose en un movimiento ciudadano con una dimensión más abierta y amplia que la que permitía la estructura de las asociaciones de vecinos en aquel momento. $\mathrm{Y}$ es en este nuevo contexto de una plataforma ciudadana en el que Portes Obertes pudo desarrollarse y adquirir un carácter muy particular.

La primera cuestión que se planteó fue la carencia de financiación e infraestructura para desarrollar el proyecto, por lo que la organización y definición del mismo debía ser el resultado de esta situación. En cuanto a la organización podíamos aportar nuestros conocimientos y la experiencia en la organización de un evento multitudinario como fue Movimiento-Inercia pero necesitábamos que fueran los propios vecinos los cogestores del mismo, aportando su capacidad relacional con el barrio y adquiriendo algunas herramien-

1. http://www.upv.es/inercia/fichinsc.html [consulta el 4/11/2015]. 
tas de gestión y de aproximación a las prácticas artísticas contemporáneas. En la definición del evento las carencias de financiación e infraestructura y la imposibilidad de ayuda por parte de las instituciones a las que estábamos enfrentados, sólo podían ser sustituidas por la aportación de recursos propios, y ¿cuáles son esos recursos para un grupo de vecinos de un barrio humilde de clase trabajadora? Sus propias casas y las calles de su barrio, así pues nos encontramos con unas magníficas y exclusivas instalaciones expositivas. ¿Qué podíamos ofrecer a los artistas participantes en estas condiciones? El tratamiento respetuoso de su obra, una cuidada instalación, su difusión, inicialmente a través de la incipiente internet, hasta que llegado el momento encontramos la forma de autofinanciar las publicaciones de cada edición, que se convirtieron en un instrumento más de difusión tanto del evento como de la problemática, un sofá en el que descansar o pasar un tiempo en el barrio y nevera, todo ello aportado por los vecinos. La posibilidad de pagar desplazamientos o un caché por el trabajo de los artistas no era posible por razones obvias. Sin duda, la solidaridad y el compromiso de los artistas con el Cabanyal desde el primer momento fueron esenciales para todo el proyecto.

Cabanyal Portes Obertes se planteó como un evento implicado con una problemática urbana, y por lo tanto debía plantear unos objetivos de mayor alcance que la propia exposición de los trabajos artísticos, en este sentido desde un primer momento planteamos cuáles eran estos objetivos, tal y como expusimos en el texto de introducción de la primera publicación que pudimos realizar del evento en 2003 y que recogía las ediciones de los años anteriores desde 1998: "Al tiempo planteamos que este evento tiene unos objetivos que deben ser claramente definidos. La sensibilización en torno a esta problemática debe alcanzar al mayor número de personas posible y la muestra debe ser un altavoz que la amplifique en el contexto de la ciudad, y fuera de ella, rompiendo cierta voluntad de minimizarla por parte de las autoridades locales del momento. Generando una imagen que muestre la verdadera complejidad y gravedad de esta situación frente a la instrumentalización mediática de los promotores del proyecto. En segundo lugar, debe actuar sobre los propios vecinos del barrio motivando su participación, reactivando sus elementos identitarios, un cierto orgullo de ser de barrios, durante tanto tiempo olvidados por las administraciones políticas que los han abandonado a su suerte, haciendo dejación de sus obligaciones sociales e incluso legales, convertidas 
en papel mojado al libre albedrío de la voluntad y en este caso de la falta de voluntad de la administración». ${ }^{2}$

También consideramos importante realizar una valoración de los resultados obtenidos y del cumplimiento de los objetivos planteados en cada momento tal y como hicimos en «Cabanyal Portes Obertes, un proyecto de intervenciones artísticas, política y urbanismo» con motivo de la presentación del proyecto en la Bienal de Arquitectura de la Habana en 2000. En esta valoración, aparecen conceptos que resultarán determinantes en estas prácticas en la actualidad: la capacidad de movilización desde el mundo de la cultura, la inserción de las prácticas artísticas en los contextos sociales, la capacidad de las prácticas artísticas en el empoderamiento social, la movilización a partir de los afectos, la experiencia lúdica como herramienta para enfrentar determinados problemas sociales. Que expresábamos de la siguiente manera:

«Con los siguientes objetivos:

- movilizar al mundo de la culturas frente a un atentado contra el patrimonio cultural de nuestro pueblo

- dar a conocer al máximo número de personas la naturaleza de este problema

- utilizar los medios de comunicación para su difusión

- generar un debate desde las artes plásticas sobre la relación del arte con la política

- crear un lugar de acción permanente donde las propuestas artísticas se inserten en la propia sociedad en la que vivimos

El método utilizado fue:

- plantear una convocatoria abierta para que los artistas que quisieran participar pudieran manifestarse sobre este tema o mostrar su solidaridad con los vecinos del barrio

2. Martínez Arroyo, E. (2003): «Cabanyal Portes Obertes. Activismo y lucha social», Cabanyal Portes Obertes, Art, política i participació ciutadana, Plataforma Salvem el Cabanyal, Valencia. Disponible en: <http://espai214.org/emiliomartinez/pag\%20 inicial.html $>$ [consulta el 5/11/2015]. 
- convocar a los vecinos del barrio para que expongan en sus casas los trabajos de los artistas y el público pueda visitarlas

- que los visitantes de la exposición conozcan el barrio, a sus habitantes y sus formas de vida y aprecien los valores que merecieron que fuera declarado Bien de Interés Cultural

- en cuanto a la financiación buscamos la participación de todos los participantes, vecinos y artistas, los últimos exponiendo su trabajo y buscando la financiación para su producción. Los vecinos prestando sus casa, vendiendo camisetas, carteles, lotería, que permitieran un mínimo de dinero para pagar los gastos que surgían en la organización del mismo y todo el mundo poniendo el tiempo que fuera necesario para que el evento pudiera realizarse con el mayor éxito posible

Los resultados obtenidos fueron:

- una gran participación de artistas y vecinos. En la primera edición de 1998 participaron 157 artistas y 27 casas particulares. En la edición de 1999 participaron 322 artistas y 49 casas particulares. Además se utilizaron los espacios de la calle, la playa, plazas y teatros del barrio, donde se realizaron obras de diferentes manifestaciones artísticas: escultura, pintura, música fotografía, teatro, cine, cómic, performances, etc.. Observando que en cada edición aumenta el número de participantes del ámbito nacional e internacional

- una enorme afluencia de público, aproximadamente 5000 personas en la primera edición y más de diez mil en la segunda, que bien por curiosidad, o por solidaridad, visitaron la exposición y el barrio

- una muy importante repercusión en los medios de comunicación (periódicos, revistas, radio y televisión) Todo ello a pesar de las restricciones impuestas en aquellos medios dominados por el partido político en el poder concretamente en el canal de la televisión pública valenciana, se impido cualquier referencia a las motivaciones que habian dado lugar a este acontecimiento, permitiendo que únicamente se hablara del aspecto «cultural»

Podemos hablar de otro resultado quizás menos evidente en un primer momento pero igualmente importante

- la valoración de los habitantes del propio barrio de lo que tienen al ver el interés que despierta en los visitantes de fuera, encontrar ese pequeño pero no menos importante orgullo de ser de un lugar 
- la calidad de los trabajos expuestos y especialmente la dedicación de aquellos artistas que realizaron un trabajo expresamente que trataba sobre la problemática del barrio

- la toma de conciencia de todos los participantes del problema que estaba sobre la mesa

- la enorme capacidad de organización de un grupo de vecinos que no están habituados a organizar un evento de este tipo

- la relación que se estableció entre vecinos y artistas, a partir del dialogo de las propuestas artísticas con un lugar concreto, con una sociedad de caras propias, nada que ver con los anonimatos de galerías y museos

- una forma lúdica de entender la protesta ciudadana, divertida en ocasiones, dramática otras pero en cualquier caso viva» ${ }^{3}$

Desde 1998 hasta 2014 se han sucedido de forma anual las ediciones de Cabanyal Portes Obertes, con el mismo carácter reivindicativo y con temáticas y formatos que han ido variando a lo largo del tiempo. La última de ellas llevaba por título «Cabanyal Portes obertes. L'art de resistir», parafraseando el proyecto «Multitud Singular: el arte de resistir», organizado en el Centro de Arte Reina Sofía de Madrid en 2009 que revisaba «las relaciones entre arte y política según la configuración de la sociedad actual. Multitud Singular pretende asomarse a nuevas formas de pensamiento sobre las ideas de revolución y de activismo». ${ }^{4} \mathrm{Y}$ que nos pareció muy pertinente para hacer un balance de buena parte de los resultados obtenidos por el proyecto a lo largo de estos años, que si bien en el Reina Sofía se planteaba desde una perspectiva global, pensábamos que sería muy pertinente desde nuestra perspectiva particular. Con la esperanza de que ésta fuera la última edición pues como habíamos anunciado repetidamente, el principal afán de Cabanyal Portes Obertes es que el proyecto de destrucción que pesaba sobre el barrio del Cabanyal de

3. Martínez Arroyo, E.; Domench Ibañez, M. (2015): «Cabanyal Portes Obertes, un proyecto de intervenciones artísticas, política y urbanismo». Disponible en $<$ http:// espai214.org/emiliomartinez/pag\%20inicial.html> [consulta el 5/11/2015].

4. http://www.museoreinasofia.es/actividades/multitud-singular-arte-resistir [consulta el $5 / 11 / 2015]$. 
Valencia desapareciera y por lo tanto Portes Obertes dejaría de tener sentido, tal y como estaba planteado desde el inicio.

Final anticipado por los reveses que el proyecto urbanístico había tenido en las instancias judiciales y en la propia opinión pública y por los síntomas de un agotamiento en el modelo de gestión municipal que había mantenido al Partido Popular en el Ayuntamiento de Valencia en las últimas décadas. Esto último se confirmó en las elecciones municipales de 2015, con una nueva mayoría progresista en el Ayuntamiento que procedió oficialmente a derogar el proyecto y afirmar su compromiso con la rehabilitación del Cabanyal tal y como veníamos demandando desde la plataforma Salvem el Cabanyal. En esta nueva situación podemos dar por concluido Cabanyal Portes Obertes.

Se acabó, ¿y ahora qué?, la incógnita que tenemos por delante tiene que ver con la situación actual del barrio, en un proceso de degradación máxima y con una futura rehabilitación por iniciar y por resolver, y muchas otras cuestiones pendientes. En este contexto, ¿cuál es la posición de las prácticas artísticas, qué papel pueden desempeñar? Cabanyal Portes Obertes siempre tuvo un carácter crítico frente a las políticas autoritarias y al modelo de ciudad propuesto que se significaba en el barrio de manera paradigmática, pero con el tiempo desarrollamos proyectos paralelos con un enfoque distinto que deberíamos atender porque creemos que son propuestas interesantes de futuro, Cabanyal Arxiu Viu y CraftCabanyal.

Portes Obertes centró buena parte de sus esfuerzos en romper la dinámica de los medios respecto al problema urbanístico para introducir las reivindicaciones ciudadanas por la defensa del patrimonio histórico y social del Cabanyal. Cabanyal Arxiu Viu y CraftCabanyal son proyectos en los que la defensa de la identidad del lugar, histórico y social, la diferencia y complejidad de los modelos de vida que aparecen en la ciudad contemporánea como valores a proteger frente a las tendencias globalizadoras de la ciudad uniforme que avanza desde algunas posiciones neoliberales en las que todos los órdenes se subordinan a la dimensión económica de la inmediatez. Estos proyectos inciden en una forma de pensar la ciudad a un medio y largo plazo en el que la pervivencia de identidades locales propias es una garantía de futuro y diversidad. Las prácticas artísticas de carácter político evolucionan en prácticas de carácter colaborativo ciudadano en las que la dimensión crítica permanece 
y se centra sobre las relaciones interpersonales, sobre los individuos y las colectividades, sobre los afectos con las personas y con los lugares.

Cabanyal Arxiu Viu y CraftCabanyal son dos planteamientos desde posiciones muy diferentes, Cabanyal Arxiu Viu ${ }^{5}$ es un proyecto en el que colaboramos con la Asociación la Esfera Azul, en 2011, que contó con la ayuda del Ministerio de Cultura, y que recibió el reconocimiento de los premios que Europa Nostra otorga anualmente en la defensa del patrimonio en la categoría de Educación, formación y sensibilización. Iniciado en 2011 se diseñó para que pudiera desarrollarse en el tiempo a través de un archivo en internet que permitiera la actualización continua de sus contenidos, está en activo en estos momentos. CraftCabanyal propuesto por la artista Bia Santos a la Plataforma Salvem el Cabanyal, está gestionado y se mantiene por la colaboración del colectivo que participa en sus acciones y propone una herramienta muy útil para mantener una posición de visibilización en el espacio público y en las redes sociales de los vecinos y vecinas del barrio que siguen revindicando la rehabilitación del Cabanyal frente a las instituciones y los poderes públicos: «Es un trabajo colectivo de craftivismo, que genera obras de artes participativas, realizadas por artistas de tres generaciones de vecinos, vecinas y amantes del Cabanyal, que utilizamos los valores de los trabajos realizados en general en la esfera privada como el bordado, el crochet, patchwork, el ganchillo, etc. que pasan a ser vehículo de protesta en relación a la problemática del barrio del Cabanyal». ${ }^{6}$

Cabanyal Arxiu Viu y CraftCabanyal son proyectos en desarrollo que plantean la posibilidad de seguir un trabajo colaborativo y crítico en la esfera

5. «Cabanyal Archivo Vivo es un proyecto propuesto por la asociación La Esfera Azul, coordinado por Lupe Frigols, Emilio Martinez y Bia Santos, que tiene como objetivo la puesta en valor, a través de las herramientas de la cultura, de los valores, la identidad, la memoria y el patrimonio del barrio del Cabanyal en Valencia, amenazado por los proyectos urbanísticos que pesan sobre él en la última década.

Inicialmente, Cabanyal Archivo Vivo es un conjunto de acciones en el espacio real y en internet, que abarcan el ámbito pedagógico (con la realización de un material sobre el patrimonio arquitectónico y cultural del barrio), social (encuentros y mesas redondas con especialistas y asociaciones vecinales del litoral marítimo) y proyectos artísticos (una serie de propuestas a partir de conceptos de geolocalización y la edición de un número especial de la revista La más bella).» Disponible en $<$ http://www. cabanyalarchivovivo.es/que_es_archivo.html> [consulta el 5/11/2015].

6. http://craftcabanyal.blogspot.com.es/ [consulta el 5/11/2015]. 
pública, se desarrollaron en paralelo a Cabanyal Portes Obertes. CraftCabanyal realizó sus primeras acciones en la XV edición, 2013. Ambos han iniciado una andadura propia y representan una posibilidad real de actuar en el contexto de la ciudad contemporánea.

\section{Referencias}

Martínez Arroyo, E. (2003): «Cabanyal Portes Obertes. Activismo y lucha social», Cabanyal Portes Obertes, Art, política i participació ciutadana, Plataforma Salvem el Cabanyal, Valencia. Disponible en: $<$ http://espai214.org/emiliomartinez/pag\%20inicial.html $>$ [consulta el 5/11/2015].

- y Domenech Ibañez, M. (2015): «Cabanyal Portes Obertes, un proyecto de intervenciones artísticas, política y urbanismo». Disponible en $<$ http://espai214.org/emiliomartinez/pag\%20inicial.html $>$ [consulta el 5/11/2015]. 
